\title{
Influence-Aware Truth Discovery
}

\author{
Hengtong Zhang, Qi Li, Fenglong Ma, Houping Xiao, Yaliang Li, Jing Gao, Lu Su \\ SUNY Buffalo, Buffalo, NY USA \\ \{hengtong, qli22, fenglong, houpingx, yaliangl, jing, lusu\}@buffalo.edu
}

\begin{abstract}
In the age of big data, information for the same entity can be obtained from different sources, which is inevitably conflicting. Therefore, aggregation methods are needed to identify the trustworthy information from such conflicting data. Truth discovery, which improves the aggregation results by estimating source trustworthiness and discovering truths simultaneously, has become an emerging field. Most truth discovery methods assume that sources make their claims independently, which may not be true in practice. As a matter of fact, influences among sources are ubiquitous and the claims made by one source may be influenced by others. Although there is some work that considers source correlation, those methods are designed to handle categorical claims, which is not general enough to represent the complicated real world applications. To tackle these challenges in truth discovery, we propose an unsupervised probabilistic model named IATD. The model takes source correlations as prior for influence derivation. To model influences among sources, we introduce "claim trustworthiness", which fuses the trustworthiness of the source which provides the claim and the trustworthiness of its influencers. Besides, the proposed model can handle different data types using different distributions in the probabilistic model. Experiments on real-world datasets show that IATD model can improve the aggregation performance compared with the state-of-the-art truth discovery approaches. The properties of IATD model are further illustrated using simulated datasets.
\end{abstract}

\section{CCS Concepts}

-Information systems $\rightarrow$ Data mining;

\section{Keywords}

Truth discovery; unsupervised learning; probabilistic model

Permission to make digital or hard copies of all or part of this work for personal or classroom use is granted without fee provided that copies are not made or distributed for profit or commercial advantage and that copies bear this notice and the full citation on the first page. Copyrights for components of this work owned by others than ACM must be honored. Abstracting with credit is permitted. To copy otherwise, or republish, to post on servers or to redistribute to lists, requires prior specific permission and/or a fee. Request permissions from permissions@ acm.org.

CIKM'16, October 24-28, 2016, Indianapolis, IN, USA

(c) 2016 ACM. ISBN 978-1-4503-4073-1/16/10 . \$ $\$ 15.00$

DOI: http://dx.doi.org/10.1145/2983323.2983785

\section{INTRODUCTION}

The past decades have witnessed an explosion of data collected from a variety of channels, such as web-scale search engines, crowd-sourcing platforms, and social media platforms. Integrating data from disparate sources can lead to novel insights in scientific, industrial, and governmental domains. However, claims about the same entity may conflict each other due to recording errors, noise, machine failures, malicious attacks, etc. Therefore, to get the most trustworthy information (i.e. the true facts), the aggregation of multi-source data needs to be applied so that the noise from individual sources can be mitigated.

Intuitively, the most straightforward aggregation approach is majority voting, which treats the claims from the majority of the sources as the truth. However, the major drawback of majority voting is that all sources are treated as equally reliable. Consequently, when there exists a large amount of low-quality sources, majority voting may lead to unsatisfactory performance. To tackle this problem, truth discovery, which aims to estimate the true facts from conflicting data sources by considering source trustworthiness, has received lots of attention [1-3,6-10,12,14,15, 18,21,22]. Many truth discovery approaches are developed based on two intuitive principles: (1) If a source provides many true claims, the trustworthiness of the source is high. (2) If a claim is supported by many trustworthy sources, this claim is likely to be true. Based on such principles, truth discovery methods tightly combine truth computation and source trustworthiness estimation to provide more accurate aggregation results.

To simplify the design, many existing truth discovery methods make an assumption that data sources provide their claims independently. However, in real life, explicit and implicit influences among sources are ubiquitous, which makes this assumption invalid. For example, on social media platforms, a person can be easily influenced by others when he/she makes a claim towards an entity or an event. Therefore, the claim may come from not only his/her own knowledge but also the knowledge of his/her friends. In such cases, the provided claims are no longer independent. When estimating the true facts, we need to consider the trustworthiness of the source itself and its related sources. If the influences among sources are ignored and the provided claims are treated as independent ones, it is likely that the estimation of source trustworthiness is inaccurate, and thus the performance of truth discovery is degraded. Therefore, it is crucial to take these inter-source influences into consideration to more accurately estimate source trustworthiness for 
truth discovery tasks. There is some truth discovery work that considers source correlations $[2,19]$. However, these methods are limited in the data types that can be applied to. Specifically, they all treat claims as categorical, which cannot represent the complicated real-world applications. As shown in $[6,7,25]$, numerical data and heterogeneous data are also common in truth discovery problems, and treating them as categorical data is inappropriate.

To tackle these challenges, we propose a novel approach named Influence- $\underline{\mathbf{A} w a r e} \underline{\text { Truth }} \underline{\text { Discovery }}($ IATD), an unsupervised full Bayesian model which can utilize the preknown source correlations as the prior. Different from existing truth discovery work, IATD introduces the concept of "claim trustworthiness", which fuses the trustworthiness of the source which provides the claim and the trustworthiness of its influencers by an ensemble parameter. Such a design enables us to precisely model the degree of inter-source influences as well as their relations towards source trustworthiness estimation. Using different distributions, we manage to utilize the fused "claim trustworthiness" for the generation of claims which are of different data types.

The advantages of the proposed IATD model are as follows. Firstly, by considering the ubiquitous source correlations, such as social ties and network relationships, the proposed model outperforms the state-of-the-art truth discovery approaches. Secondly, the model can explicitly estimate the trustworthiness for every claim, which is obtained by considering inter-source influences and claim generation in an interpretable way. Last but not least, the proposed IATD model can handle both numerical and categorical data types, and thus can be applied to a large variety of real-world scenarios.

Experiments on real-world datasets demonstrate that the proposed IATD model can improve the performance of the truth estimation compared with other state-of-the-art truth discovery approaches. Further, to comprehensively show the insights of the proposed IATD model, we conduct experiments on simulated datasets, where source correlation and contexts can be controlled. Using these simulations, we illustrate the behavior of the proposed IATD model under various conditions.

Contributions: It is worthwhile to highlight the major contributions of this paper:

- We recognize the importance of modeling source relations as prior knowledge in truth discovery tasks. We also consider to handle different data types in modeling truth discovery with source correlations.

- We propose a probabilistic model that incorporates source correlations to simultaneously learn trustworthiness for each source and each claim, as well as the true claims for all entities.

- We experimentally show that the proposed IATD model outperforms existing truth discovery methods on real world datasets. We further demonstrate the characteristics of the proposed model on the simulated datasets.

This paper is organized as follows: In Section 2, we formally define the problem of "influence-aware truth discovery". Then we describe the proposed IATD model and provide a method for parameter estimation of IATD in detail in Section 3. We conduct a series of experiments and case studies on both synthetic and real-world data in Section 4 .
Section 5 is a survey of related work, and we conclude the paper in Section 6.

\section{PROBLEM DEFINITION}

Before describing our proposed model, we start by introducing some terminologies, followed by the formal problem definition.

Definition 1. An entity is an item of interest. The set of $M$ entities is denoted as $V=\{v\}_{1}^{M}$.

Definition 2. A source is the place where information about entities is collected. The set of sources is denoted as $S=\{s\}_{1}^{N}$.

Definition 3. A claim is defined as a piece of information provided by a source towards an entity, and the set of claims is denoted as $C$. We use $C \cdot, v$ to denote the set of claims for entity $v$, and $c_{s, v}$ denotes the claim from source $s$ on entity $v$.

Definition 4. A truth is the most trustworthy piece of information for an entity. The set of truths is denoted as $T=\{t\}_{1}^{M}$.

Definition 5. An influence set $A_{s, v}$ is a set of sources that may influence source $s$, when s makes a claim on entity $v$.

Definition 6. (PRoblem Definition) Given a set of sources $S=\{s\}_{1}^{N}$, a set of entities $V=\{v\}_{1}^{M}$, a set of claims $C_{s=1, v=1}^{N, M}$ and a set of influencers for every claim $A_{s=1, v=1}^{N, M}$, the goal of our proposed model is to learn the estimated truths for entities $T=\{t\}_{1}^{M}$ as well as the trustworthiness for the sources.

Note: In this paper, we only consider the single truth scenario, i.e., for each entity, there is only one truth.

\section{METHODOLOGY}

In this section, we describe the IATD model in details. We first provide a high-level overview of the proposed model. This is followed by a detailed mathematical specification. Finally, we provide a comprehensive description of the model fitting procedure based on Expectation Maximization.

\subsection{Overview}

The IATD model specifies a two-stage generative process of claims. The first stage specifies the generation of sources' individual trustworthiness as well as the influenceaware trustworthiness fusion given the influencer set for each claim. The second stage specifies the generation of heterogeneous claims, given the "claim trustworthiness" of each claim. Here, we describe the intuition behind the modeling before detailing the IATD model.

Trustworthiness Generation: According to our investigation of the real world data, we find that source's final decisions are usually based on the combination of its own trustworthiness and its trusted sources' trustworthiness. A source may make its claims based on its own trustworthiness, but may be influenced by the sources it trusts at the same time. In our model, we introduce the "claim trustworthiness", which fuses the trustworthiness of current source 
Table 1: Notations

\begin{tabular}{c|l}
\hline Notations & Meaning \\
\hline \hline$N$ & the number of sources in the dataset \\
\hline$\lambda$ & the number of entities in the dataset \\
\hline$\sigma_{s}$ & global influence smoothing parameter \\
\hline$b_{v}$ & individual source deviation for source $s$ \\
\hline$g^{(c)}, g^{(n)}$ & $\begin{array}{l}\text { pre-tuned global deviation bias for categor- } \\
\text { ical and numerical data respectively }\end{array}$ \\
\hline$\gamma_{v}$ & $\begin{array}{l}\text { the number of possible discrete values for } \\
\text { entity } v \text { with categorical claims }\end{array}$ \\
\hline$C_{s, \cdot}$ & the set of claims given by source $s$ \\
\hline$C_{\cdot, v}$ & the set of claims on entity $v$ \\
\hline$c_{s, v}$ & the claim given by source $s$ on entity $v$ \\
\hline$\epsilon_{v}$ & estimated truth of entity $v$ \\
\hline$A_{s, v}$ & $\begin{array}{l}\text { the deviation value of the claim given by } \\
\text { source } s \text { on entity } v\end{array}$ \\
\hline$\alpha_{e}, \beta_{e}$ & $\begin{array}{l}\text { the set of sources which may influence } s, \\
\text { when making claim } c_{s, v}\end{array}$ \\
\hline$\mu_{b}, \sigma_{b}^{2}$ & $\begin{array}{l}\text { parameters for Inverse-Gamma prior of } \\
\text { source deviation }\end{array}$ \\
\hline$\mu_{t}, \sigma_{t}^{2}$ & $\begin{array}{l}\text { parameters for Gaussian prior of entity- } \\
\text { specific difficulty variable } \\
\text { value of numerical entities }\end{array}$ \\
\hline
\end{tabular}

and the trustworthiness of potential influencers linearly by an ensemble parameter. This can better explain the phenomenon when the credibility of multiple claims from a single source is inconsistent sometime. Moreover, in our model, if a source makes true claims for some entities, the trustworthiness of this source and its influencers will be increased. On the contrary, if a source makes false claims for some entities, this source and its influencers will all suffer a decrease in their trustworthiness. In this paper, in order to fit both categorical and numerical data, we evaluate the source trustworthiness using a variable $\sigma$, which models the claim deviation tendency of a source. The value of $\sigma$ is inversely proportional to the trustworthiness degree of a single source.

Truth Generation: In IATD, we discuss two typical types of entities, i.e. categorical and numerical. For entities with categorical claims, the true values are modeled as random variables following uniform distributions, as we assume there is a single true value for each entity and the false values should be uniformly distributed. For entities with numerical claims, we model the true values as a random variable following Gaussian distributions. These distributions are commonly used to model categorical and numerical data, respectively.

Claim Generation: Once we get the claim trustworthiness and truth for each claim, we can utilize these variables to model the generation process for claims. For both types of claims, we assume that the claim from a source is associated with: (1) the claim deviation, and (2) the difficulty of the entity. For entities with categorical claims, we consider the posterior of a claim to be true, given the estimated truth. If the claim deviation is high, the probability of the claim being true should be small, and vice versa. For entities with numerical claims, we model the generation of claim

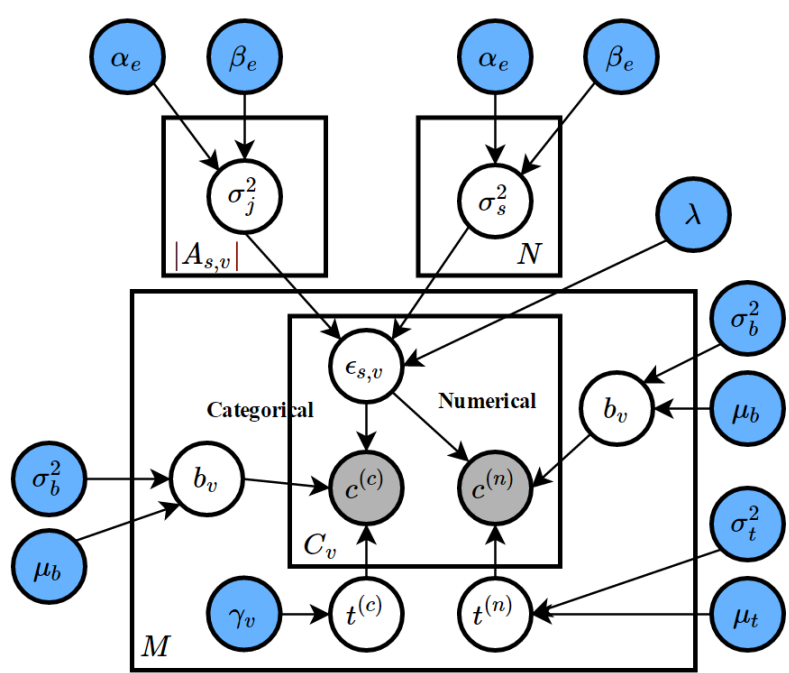

Figure 1: Plate notation for the proposed IATD Model. In the graph, blue circles denote the hyper-parameters, white circle denote the latent variables, and gray circles stand for the claims. $c^{(c)}$ stands for categorical claims, while $c^{(n)}$ stands for numerical claims.

using a Gaussian distribution, whose mean is the truth, and the variance parameter is the claim deviation. This indicates that high trustworthiness claims have smaller deviations from the truth. Moreover, for different entities, the difficulty of obtaining true claims may differ. Therefore, we introduce the entity-specific difficulty parameter to model this phenomenon.

Fig. 1 shows the graphical structure of the model, and Table 1 lists the descriptions of the variables. The generative process is shown as follows:

- For the $s$-th source $(s=1,2, \cdots, N)$

- Draw $\sigma_{s}^{2} \sim \Gamma^{-1}\left(\alpha_{e}, \beta_{e}\right)$.

- For the $v$-th entity $(v=1,2, \cdots, M)$

- For an entity with categorical claims $v$, draw a true fact $t_{v} \sim \mathrm{U}\left(\gamma_{v}\right)$.

- For an entity with numerical claims $v$, draw a true fact $t_{v} \sim \mathcal{N}\left(\mu_{t}, \sigma_{t}^{2}\right)$.

- For each claim $c_{s, v}$

- For an entity with categorical claims $v$, draw a claim $c_{s, v} \sim \operatorname{logistic}\left(-\epsilon_{s, v}+b_{v}+g^{(c)}\right)$.

- For an entity with numerical claims $v$, draw a claim $c_{s, v} \sim \mathcal{N}\left(t_{v},\left(\epsilon_{s, v}+b_{v}+g^{(n)}\right)^{2}\right)$.

\subsection{Model Specification}

In this section, we provide a detailed description of the proposed model. We first specify the generation of sources' individual trustworthiness and "influence-aware trustworthiness fusion". Then we describe the generation of different claims separately based on claim trustworthiness. 


\subsubsection{Trustworthiness Modeling}

The source and claim trustworthiness are modeled via deviation variables $\sigma$ as follows. For each source $s$, we draw $\sigma_{s}^{2}$ from an Inverse-Gamma distribution with hyper-parameters $\left(\alpha_{e}, \beta_{e}\right)$, where $\alpha_{e}$ and $\beta_{e}$ are shape and rate parameters respectively. Therefore,

$$
\sigma_{s}^{2} \sim \Gamma^{-1}\left(\alpha_{e}, \beta_{e}\right) \propto\left(\sigma_{s}^{2}\right)^{-\alpha_{e}-1} \exp \left(-\beta_{e} \sigma_{s}^{-2}\right) .
$$

Mathematically, $\sigma_{s}^{2}$ is the variance variable for Gaussian distribution and the value of $\sigma_{s}^{2}$ is inversely proportional to the trustworthiness of a source. When parameter $\alpha_{e}>1$ the Inverse-Gamma distribution concentrates mostly around its mode $\frac{\beta_{e}}{\alpha_{e}+1}$. This generally means that the deviation of most sources should be around a certain value and there are a few with much higher or lower deviation in our assumption.

Given the definition of individual deviation for every source, we can further model the phenomenon that a source $s$ gets influenced by others when generating claims for entity $v$. To model the claim deviation, we introduce an auxiliary variable $\epsilon_{s, v}$, which denotes the deviation of source $s$ when it offers a claim on entity $v$ (i.e. claim deviation). This variable reflects both the deviation of the source itself and the deviation of sources it relates. Let $\sigma_{s}$ and $\sigma_{j}$ denote the source deviation of $s$ and $j$, and $A_{s, v}$ be the set of sources that may influence $s$ when $s$ makes a claim on entity $v$. We can model the trustworthiness fusion as follows:

1. If we can infer that a user is influenced by others, then $\epsilon_{s, v}$ is defined as:

$$
\epsilon_{s, v}=\frac{\lambda}{\left|A_{s, v}\right|} \sum_{j \in A_{s, v}} \sigma_{j}+(1-\lambda) \cdot \sigma_{s} .
$$

2. If there is no evidence that the source is influenced (i.e. $\left.A_{s, v}=\Phi\right)$, then

$$
\epsilon_{s, v}=\sigma_{s}
$$

Note that the value of the auxiliary variable $\epsilon$ can be calculated directly given two deviation variables $\sigma_{s}$ and $\sigma_{j}$. Therefore, it does not lead to the increase the number of parameters.

Further, as the difficulties of obtaining the value of entities may be different, we introduce an entity-specific bias variable $b_{v}$ to make some adjustments. $b_{v}$ is drawn from a Gaussian distribution:

$$
b_{v} \sim \mathcal{N}\left(\mu_{b}, \sigma_{b}^{2}\right),
$$

where $\mu_{b}$ and $\sigma_{b}^{2}$ are the parameters of the Gaussian distribution.

\subsubsection{Claim Modeling}

Given the claim trustworthiness $\epsilon_{s, v}$ and entity-specific bias $b_{v}$, we now describe the posterior probability of observed claims on entity $v$ from the source $s$, i.e., $c_{s, v}$, given the latent true fact $t_{v}$. The intuition is straightforward: the sources of low deviation often provide more trustworthy claims. Since the claim generation process for categorical and numerical claims are different, we handle them using different formulations.

Categorical Claim Modeling: For categorical claims, we model the probability of a claim $c_{s, v}$ being true using a Bernoulli distribution. Intuitively, the probability that source $s$ offers a true claim to entity $v$ relies on: (1) the claim deviation, and (2) the difficulty of obtaining the true value of the entity. Specifically, the probability is defined as:

$$
p\left(c_{s, v}=x \mid t_{v}=x, \epsilon_{s, v}, b_{v}\right)=h\left(-\epsilon_{s, v}+b_{v}+g^{(c)}\right),
$$

where $h(\cdot)$ is a logistic function, and $g^{(c)}$ is a pre-tuned global bias for entities with categorical claims. We can see that if the claim deviation $\epsilon_{s, v}$ is small, the probability of $c_{s, v}$ being a true claim is large, and vise versa.

We assume the probability that source $s$ offers an incorrect claim to entity $v$ is from a Uniform distribution, so the probability that source $s$ offers an incorrect claim to entity $v$ is modeled as:

$$
p\left(c_{s, v} \neq x \mid t_{v}=x, \epsilon_{s, v}, b_{v}\right)=\frac{1-h\left(-\epsilon_{s, v}+b_{v}+g^{(c)}\right)}{\gamma_{v}-1} .
$$

Combining Eq. (5) and Eq. (6), we can get the probability that source $s$ makes the claim $c_{s, v}$, given the claim deviation $\epsilon_{s, v}$, the truth estimation $t_{v}$, and the entity-specific bias variable $b_{v}$ :

$$
\begin{aligned}
& p\left(c_{s, v} \mid t_{v}=x, \epsilon_{s, v}, b_{v}\right) \\
= & h\left(-\epsilon_{s, v}+b_{v}+g^{(c)}\right)^{\delta\left(c_{s, v}, t_{v}\right)} \\
& \times\left(\frac{1-h\left(-\epsilon_{s, v}+b_{v}+g^{(c)}\right)}{\gamma_{v}-1}\right)^{1-\delta\left(c_{s, v}, t_{v}\right)},
\end{aligned}
$$

where $\delta(\cdot, \cdot)$ is the Kronecker delta function.

Numerical Claim Modeling: For entities with numerical claims, we draw a true claim from a Gaussian distribution

$$
t_{v} \sim \mathcal{N}\left(\mu_{t}, \sigma_{t}^{2}\right)
$$

where $\mu_{t}$ controls the prior estimate of the truth and $\sigma_{t}^{2}$ captures the prior deviation. The probability of source $s$ offering a correct claim to entity $v$ is modeled as a Gaussian distribution with the mean of the estimated truth $t_{v}$, and the deviation of $\left(\epsilon_{s, v}+b_{v}+g^{(n)}\right)^{2}$, i.e.,

$$
\begin{aligned}
& p\left(c_{s, v} \mid t_{v}=x, \epsilon_{s, v}, b_{v}\right) \sim \mathcal{N}\left(t_{v},\left(\epsilon_{s, v}+b_{v}+g^{(n)}\right)^{2}\right) \\
& \propto\left(\epsilon_{s, v}+b_{v}+g^{(n)}\right)^{-1} \exp \left(-\frac{\left(c_{s, v}-t_{v}\right)^{2}}{2\left(\epsilon_{s, v}+b_{v}+g^{(n)}\right)^{2}}\right),
\end{aligned}
$$

where $g^{(n)}$ is a pre-tuned global bias for entities with numerical claims.

The truth of entity $v$, the claim deviation $\epsilon_{s, v}$, and the $v$ 's bias variable $b_{v}$ jointly capture the precision of the claim. When claim deviation $\epsilon_{s, v}$ and/or bias variable $b_{v}$ get smaller, the claim $c_{s, v}$ should be closer to the truth, and vise versa.

\subsection{Discussion}

Here we describe the influence derivation, which can have effects on the final performance.

Let $c_{s, v}$, which is provided by source $s$ towards entity $v$, be the claim we are currently working on. Influence derivation is to determine the set of sources that potentially influence $s$ when $s$ provides claim $c_{s, v}$. For brevity, we only introduce a general and straightforward way for influence derivation. Let $A_{s, v}$ be the source set who may influence $s$, when it makes claim $c_{s, v}$. If there is a directed relation from $s$ to $j$ and they make the same false claim on entity $v$, we treat $s$ 
to be influenced by $j$, when $s$ provides its claim $c_{s, v}$, i.e.,

$$
j \in A_{s, v} \Longleftrightarrow\left\{\begin{array}{l}
\exists s \rightarrow j, \\
c_{s, v}=c_{j, v}, \\
c_{s, v} \neq \hat{t}_{v},
\end{array}\right.
$$

where $\hat{t}_{v}$ is the estimated truth for entity $v$.

Note that more sophisticated influence detection methods can be deployed here. For simplicity, we choose this approach.

\subsection{Model Fitting}

The fitting process is to estimate the value of hidden source deviation $\sigma_{s}$ and the true fact $t$ of each entity, given the set of claims and source correlations. The negative loglikelihood of observations, latent variables, and parameters given the hyper-parameters can be written as:

$$
\begin{aligned}
\mathcal{L}= & -\log \left(p\left(C, \boldsymbol{t}, \boldsymbol{\epsilon}, \boldsymbol{b} \mid \alpha_{e}, \beta_{e}, \sigma_{b}^{2}, \mu_{t}, \sigma_{t}^{2}\right)\right) \\
= & -\sum_{s=1}^{N} p\left(\sigma_{s}^{2} \mid \alpha_{e}, \beta_{e}\right)-\sum_{v=1}^{M} p\left(t_{v} \mid \mu_{t}, \sigma_{t}^{2}\right)-\sum_{v=1}^{M} p\left(b_{v} \mid \mu_{b}, \sigma_{b}^{2}\right) \\
& -\sum_{s=1}^{N} \sum_{v=1}^{M} p\left(c_{s, v} \mid \epsilon_{s, v}, t_{v}, b_{v}\right)
\end{aligned}
$$

For entities with numerical claims, Eq. (11) is formulated as:

$$
\begin{aligned}
\mathcal{L} \propto & \sum_{s=1}^{N} \sum_{v=1}^{M}\left(\frac{\left(c_{s, v}-t_{v}\right)^{2}}{2\left(b_{v}+\epsilon_{s, v}+g^{(n)}\right)^{2}}+\log \left(b_{v}+\epsilon_{s, v}+g^{(n)}\right)\right) \\
& +\sum_{s=1}^{N}\left(2\left(1+\alpha_{e}\right) \log \sigma_{s}+\beta_{e} \sigma_{s}^{-2}\right)+\sum_{v=1}^{M} \frac{\left(b_{v}-\mu_{b}\right)^{2}}{2 \sigma_{b}^{2}} \\
& +\sum_{v=1}^{M} \frac{\left(t_{v}-\mu_{t}\right)^{2}}{2 \sigma_{t}^{2}} .
\end{aligned}
$$

For entities with categorical claims, Eq. (11) is formulated as:

$$
\begin{aligned}
\mathcal{L} \propto & \sum_{s=1}^{N} \sum_{v=1}^{M}\left(-\delta\left(c_{s, v}, t_{v}\right) \cdot \log \left(h\left(-\epsilon_{s, v}+b_{v}+g^{(c)}\right)\right)\right. \\
& \left.-\left(1-\delta\left(c_{s, v}, t_{v}\right)\right) \cdot \log \left(1-h\left(-\epsilon_{s, v}+b_{v}+g^{(c)}\right)\right)\right) \\
& +\sum_{s=1}^{N}\left(2\left(1+\alpha_{e}\right) \log \sigma_{s}+\beta_{e} \sigma_{s}^{-2}\right)+\sum_{v=1}^{M} \frac{\left(b_{v}-\mu_{b}\right)^{2}}{2 \sigma_{b}^{2}} .
\end{aligned}
$$

To fit such models with latent variables, we refer to EM algorithm. In E-Step, the algorithm takes the expectation of complete data likelihood with respect to the posterior of latent variable $t$, and in M-Step it maximizes the expectation of complete data likelihood from E-Step to update model parameters $\sigma_{s}, \sigma_{j}$, and $b_{v}$.

\subsubsection{E-Step}

For entities with categorical claims, the major computational bottleneck in E-Step is that the posteriors of latent variables are not available in a closed form. Hence, we take recourse to Monte Carlo methods, which perform Gibbs sampling to randomly sample variables from their posterior distributions. Specifically, we sample $t_{v}$ as:

$$
\begin{aligned}
& p\left(t_{v}=x \mid \epsilon_{s, v}, b_{v}, g^{(c)}\right) \\
& \propto p\left(t_{v}=x\right) \prod_{s=1}^{N_{v}} p\left(c_{s, v} \mid t_{v}=x, \epsilon_{s, v}, b_{v}, g^{(c)}\right) .
\end{aligned}
$$

Eq. (14) can be calculated directly using Eq. (7).

For entities with numerical claims, we can get the closedform expression of $t_{v}$ by solving $\frac{\partial \mathcal{L}}{\partial t_{v}}=0$ using Eq. (12), which is:

$$
t_{v}=\frac{\mu_{t} \sigma_{t}^{-2}+\sum_{s=1}^{N_{v}} c_{s, v}\left(\epsilon_{s}+b_{v}+g^{(n)}\right)^{-2}}{\sigma_{t}^{-2}+\sum_{s=1}^{N_{v}}\left(\epsilon_{s}+b_{v}+g^{(n)}\right)^{-2}} .
$$

Given the value of latent variables, we can derive the expression of complete data likelihood.

\subsubsection{M-Step}

In M-Step, we need to find the parameters that maximize the likelihood computed in the E-Step. As the deviation variables $\sigma_{s}$ and $\sigma_{j}$ are above zero, the optimization problem should be formulated as:

$$
\begin{aligned}
& \min _{\sigma_{s}, \sigma_{j}, b} \mathcal{L} \\
& \text { s.t. } \sigma_{s}>0, \sigma_{j}>0 .
\end{aligned}
$$

Intuitively, we can solve that optimization objective by adapting Gradient approaches directly. However, to decrease the computation cost, we propose to solve the following optimization problem on each claim separately, which approximates the problem in Eq. (16):

$$
\begin{aligned}
& \min _{\sigma_{s}, \sigma_{j}, b} \mathcal{L}_{s, v} \\
& \text { s.t. } \sigma_{s}>0, \sigma_{j}>0 .
\end{aligned}
$$

Here, for entities with numerical claims, $\mathcal{L}_{s, v}$ is formulated as:

$$
\begin{aligned}
\mathcal{L}_{s, v} \propto & \frac{\left(c_{s, v}-t_{v}\right)^{2}}{2\left(b_{v}+\epsilon_{s, v}+g^{(n)}\right)^{2}}+\log \left(b_{v}+\epsilon_{s, v}+g^{(n)}\right) \\
& +\frac{1}{\left|C_{s, \cdot}\right|}\left(2\left(1+\alpha_{e}\right) \log \sigma_{s}+\beta_{e} \sigma_{s}^{-2}\right) \\
& +\frac{1}{\left|C_{\cdot, v}\right|} \frac{\left(b_{v}-\mu_{b}\right)^{2}}{2 \sigma_{b}^{2}}+\frac{1}{\left|C_{\cdot, v}\right|} \frac{\left(t_{v}-\mu_{t}\right)^{2}}{2 \sigma_{t}^{2}}
\end{aligned}
$$

For entities with categorical claims, $\mathcal{L}_{s, v}$ is formulated as:

$$
\begin{aligned}
\mathcal{L}_{s, v} \propto & -\delta\left(c_{s, v}, t_{v}\right) \cdot \log \left(h\left(-\epsilon_{s, v}+b_{v}+g^{(c)}\right)\right) \\
& -\left(1-\delta\left(c_{s, v}, t_{v}\right)\right) \cdot \log \left(1-h\left(-\epsilon_{s, v}+b_{v}+g^{(c)}\right)\right) \\
& +\frac{1}{\left|C_{s, \cdot}\right|}\left(2\left(1+\alpha_{e}\right) \log \sigma_{s}+\beta_{e} \sigma_{s}^{-2}\right) \\
& +\frac{1}{\left|C_{\cdot, v}\right|} \frac{\left(b_{v}-\mu_{b}\right)^{2}}{2 \sigma_{b}^{2}}
\end{aligned}
$$

It is difficult to derive the optimal closed-form solution for those variables. Therefore, we use Projection Gradient (PG) method for model fitting. PG is an extension of gradient descent method, and is commonly used for solving linearly constrained problems. In Eq. (17), the optimization problem projects $\sigma_{s}$ (or $\left.\sigma_{j}\right)$ onto $(0, \infty)$. In our implementation, $\sigma_{s}$ 
(or $\sigma_{j}$ ) is mapped as following:

$$
\mathrm{P}(\sigma)=\left\{\begin{array}{ll}
\sigma & \text { if } \sigma>10^{-5} \\
10^{-5} & \text { if } \sigma \leq 10^{-5}
\end{array} .\right.
$$

Given the projection function $P$, the update of $\sigma$ is defined by:

$$
\begin{gathered}
\sigma_{s}^{(k+1)} \leftarrow P\left(\sigma_{s}^{(k)}-\eta_{k} \frac{\partial \mathcal{L}_{s, v}}{\partial \sigma_{s}^{(k)}}\right), \\
\sigma_{j}^{(k+1)} \leftarrow P\left(\sigma_{j}^{(k)}-\eta_{k} \frac{\partial \mathcal{L}_{s, v}}{\partial \sigma_{j}^{(k)}}\right),
\end{gathered}
$$

where $P(\cdot)$ denotes the projection from $\mathbb{R}^{n}$ onto $\mathbb{R}^{+}$.

For $b_{v}$, as there is no constraint, we can simply deploy gradient descent for parameter update.

$$
b_{v}^{(k)} \leftarrow b_{v}^{(k)}-\eta_{k} \frac{\partial \mathcal{L}_{s, v}}{\partial b_{v}^{(k)}} .
$$

To speed up the PG, one crucial part is the tuning of step length $\eta_{k}$. It keeps changing in each iteration. There are a variety of strategies for searching $\eta_{k}$ and we use the algorithm in [11] (as shown in Algorithm 1). The parameters of Algorithm 1 are tuned as [11].

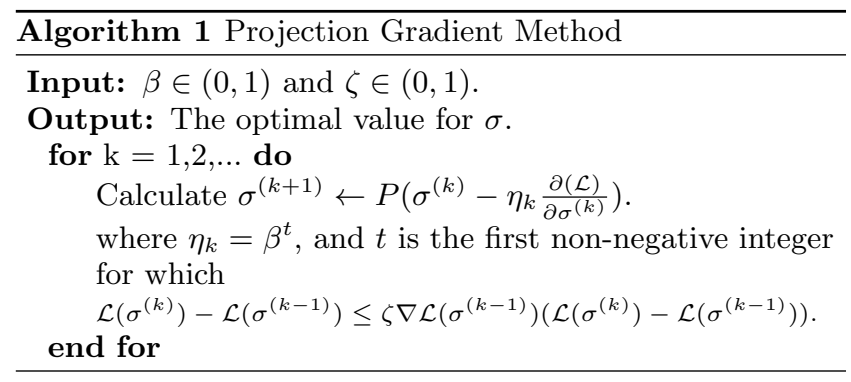

The detailed gradients of the objective with respect to the variables can be found in Appendix section.

Algorithm for Model Fitting: The overall model fitting procedure is described in Algorithm 2. We first calculate or sample an estimated truth $t_{v}$ to entity $v$ given fixed claim trustworthiness $\epsilon_{s, v}$ and entity-specific bias variable $b_{v}$. After getting $t_{v}$, we update $\sigma_{s}, \sigma_{i}$, and $b_{v}$ accordingly. The algorithm iterates between these two steps until the values of parameters and latent variables become stable.

\section{EXPERIMENTS}

In this section, we present and analyze the experimental results on both real-world and simulated data. The results show that the proposed IATD method outperforms state-ofthe-art truth discovery approaches. We first state the overall experiment settings in Section 4.1. Then we demonstrate and analyze the results on real-world and simulated data respectively.

\subsection{Experiment Settings}

Baseline Methods: To evaluate the effectiveness of the proposed model, we compare it with the following baseline methods:

- Voting: The truth estimates are obtained by the value which has the highest number of occurrences in the claim set.

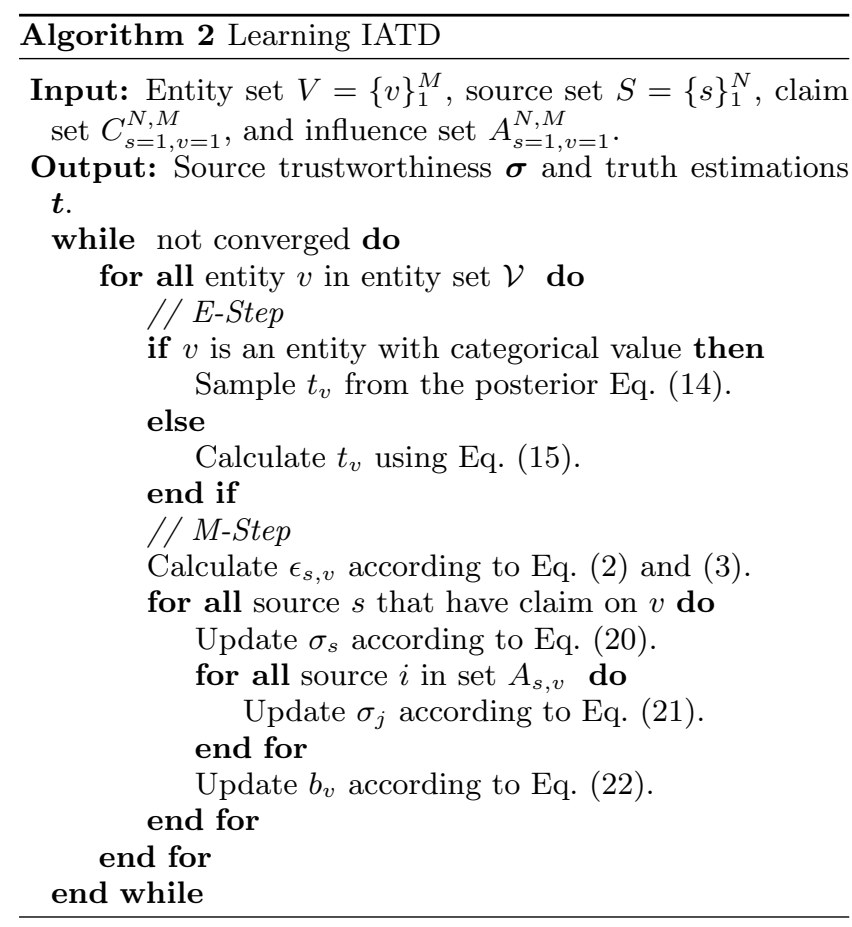

- GTM [25]: GTM is a full Bayesian approach designed for truth discovery on numerical data. ${ }^{1}$

- Invest [14]: In this method, sources distribute their reliability scores uniformly on the claims they provide, and then collect their credibility from the confidence of those claims.

- Pooled Invest [14]: This method is a variant of Invest. The difference is that the confidence of claims is linearly scaled for Pooled Invest.

- 2-Estimate [3]: 2-Estimate is an approach based on the assumption that "there is a single true value for each entity". Therefore, 2-Estimate models the complementary votes.

- 3-Estimate [3]: 3-Estimate extends 2-Estimate by considering the difficulty of obtaining the true claim for an entity.

- TruthFinder [23]: TruthFinder is a Bayesian-based approach, which computes the probability of a claim being true given the sources. Claim similarity is modeled as an implication function.

- AccuSim [2]: AccuSim is a Bayesian approach that is similar to TruthFinder. However, it considers complementary votes for claims, which is similar to 2Estimate. AccuSim also considers claim similarity.

- $C R H$ [7]: $\mathrm{CRH}$ is an optimization framework which handles different data types jointly. The goal of the optimization problem is to minimize the weighted loss of the aggregation results.

\footnotetext{
${ }^{1}$ Note: This approach is used on numerical data only, as it is incompatible with categorical data.
} 
Table 2: Statistics of the Real-world Datasets

\begin{tabular}{lcc}
\hline & Flight Dataset & Stock Dataset \\
\hline \# of Claims & $2,790,734$ & $11,748,734$ \\
\# of Entities & 204,422 & 326,423 \\
\# of Truths & 16,572 & 29,198 \\
\hline
\end{tabular}

- IATD-ni: This is a variant for the proposed IATD, which does not take inter-source influence information into consideration.

The parameters of baseline methods are set according to their authors' suggestions.

Evaluation Metrics: To evaluate the performance of truth discovery methods, two classical metrics are employed for different types of data. For both metrics, a lower value indicates a better performance.

- Error Rate: This metric is used for performance evaluation on categorical data. It is defined as the percentage of false values using an approach according to the ground truth.

- $M N A D$ : This metric is used for performance evaluation on numerical data. MAD (Mean Absolute Deviation) is a quantity on how close truth estimates are to the ground truth. As numerical data may have different scales, we normalize $M A D$ using the standard variance of each data type. $M N A D$ can be formulated as:

$$
M N A D=\frac{1}{M} \sum_{v=1}^{M} \frac{\mid t_{v}-\text { truth }_{v} \mid}{\operatorname{std}\left(c_{1, v}, c_{2, v} \cdots, c_{N, v}\right)},
$$

where $\operatorname{truth}_{v}$ stands for the ground truth for entity $v$, and the other notations are listed in Table 1.

\subsection{Experiments on Real-World Data}

Datasets: In order to evaluate the performance of the proposed model, we use two real-world datasets for experiments:

- Flight Dataset: This dataset [8] consists of 37 sources, which is collected from multiple websites. There are six different attributes in this dataset including: scheduled departure/arrival time, actual departure/arrival time, and actual departure/arrival gate. The former four attributes are numerical, while the last two attributes are categorical.

- Stock Dataset: This dataset [8] consists of 55 sources, which is collected from web search results. Specifically, Volume, Shares outstanding, and Market cap are treated as numerical data, while the other attributes are treated as categorical. The statistics of these datasets are shown in Table 2 .

The task for our experiment is to estimate the true value for each entity in these two datasets.

In our experiment, for entities with categorical claims, we set $\alpha_{e}=4.5, \beta_{e}=20, \mu_{b}=0, \sigma_{b}^{2}=1$ and $g^{(c)}=3$ for the Flight dataset, and $\alpha_{e}=10, \beta_{e}=10, \mu_{b}=0, \sigma_{b}^{2}=$ 10 and $g^{(c)}=10$ for the Stock dataset. For entities with numerical claims, we set $\alpha_{e}=4.5, \beta_{e}=100, \mu_{b}=0, \sigma_{b}^{2}=$
Table 3: Performance on Real-World Datasets

\begin{tabular}{ccccc}
\hline & \multicolumn{2}{c}{ Flight Dataset } & \multicolumn{2}{c}{ Stock Dataset } \\
Method & Error Rate & MNAD & Error Rate & MNAD \\
\hline IATD & $\mathbf{0 . 0 6 7 4}$ & $\mathbf{2 . 7 1 6 0}$ & $\mathbf{0 . 0 6 8 9}$ & 2.6734 \\
IATD-ni & 0.0795 & 2.7179 & 0.0789 & 2.6734 \\
CRH & 0.0823 & 4.8613 & 0.0700 & $\mathbf{2 . 6 4 4 5}$ \\
GTM & N/A & 7.6703 & N/A & 2.8081 \\
Voting & 0.0859 & N/A & 0.0817 & N/A \\
Invest & 0.0919 & 6.4153 & 0.0983 & 2.8081 \\
Pooled Invest & 0.0925 & 5.8562 & 0.0990 & 2.7940 \\
2-Estimate & 0.0885 & 7.4347 & 0.0726 & 2.8509 \\
3-Estimate & 0.0881 & 7.1983 & 0.0818 & 2.7749 \\
TruthFinder & 0.0950 & 8.1351 & 0.1194 & 2.7140 \\
AccuSim & 0.0881 & 7.3204 & 0.0726 & 2.8503 \\
\hline
\end{tabular}

1 and $g^{(n)}=0$ for the Flight dataset, and $\alpha_{e}=0.05, \beta_{e}=$ $0.10, \mu_{b}=0, \sigma_{b}^{2}=10$ and $g^{(n)}=0$ for the Stock dataset. $\mu_{t}$ and $\sigma_{t}^{2}$ are parameters related to the conditions of different datasets. In the preprocessing step, we shift the mean values of numerical data to 0 . Hence, $\mu_{t}$ is set to be 0 for both datasets. We set $\sigma_{t}^{2}=1$ for the Flight dataset and $\sigma_{t}^{2}=100$ for the Stock dataset.

Correlation Extraction: The Flight dataset and Stock dataset do not have explicit correlations. However, investigations in previous work indicate that there are implicit correlations among sources [8]. Therefore, we adopt a correlation extraction method based on source similarities. In this method, if two sources make many similar false claims, they are regarded to be correlated, which is one of the most important intuitions for correlation extraction. We extract the source correlations according to the claim history and calculate the Jaccard distance between each pair of sources. The similarity is defined as:

$$
\operatorname{sim}(i, j)=\frac{\left|W_{i} \cap W_{j}\right|}{\left|W_{i} \cup W_{j}\right|}=\frac{\left|W_{i} \cap W_{j}\right|}{\left|W_{i}\right|+\left|W_{j}\right|-\left|W_{i} \cap W_{j}\right|},
$$

where $W_{s}$ is the set of entities on which source $s$ makes wrong claims based on Voting. Then, we can use a threshold to determine the existence of correlations between two sources. The threshold is set to be 0.2 for the Flight dataset 0.08 for the Stock dataset. That is to say, on the Flight dataset, if $\operatorname{sim}(i, j)>0.2$, these sources are regarded as correlated. Otherwise, they are treated as independent. On the Stock dataset, if $\operatorname{sim}(i, j)>0.08$, these sources are regarded as correlated. Otherwise, they are treated as independent.

Overall Performance: The results of all the methods in terms of Error Rate and MNAD are shown in Table 3. From the table, we can see that the proposed IATD generally outperforms the baseline methods.

The reason that the proposed IATD approach can work comparable or better than other truth discovery approaches is due to the fact that influences are more precisely modeled. If we visualize the Jaccard distances between sources (Fig. 3), we can find that many sources are correlated. Therefore, the chance that these correlated sources influences each other when making claims is high. In contrast to baseline methods, the proposed IATD model takes these inter-source influences into consideration, which enables us to get more 
precise and interpretable estimates of source trustworthiness. Thus we can achieve a better performance in truth estimation.
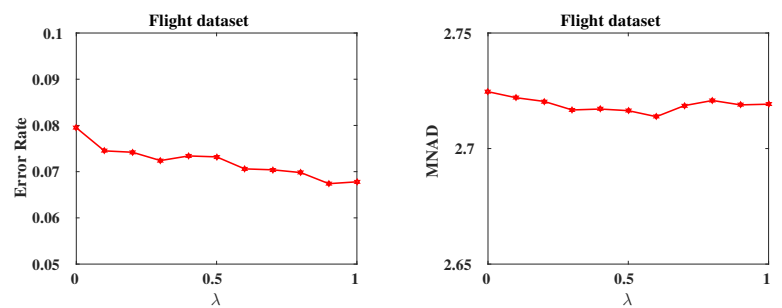

(a) Error Rate for the Flight (b) MNAD for the Flight dataset dataset
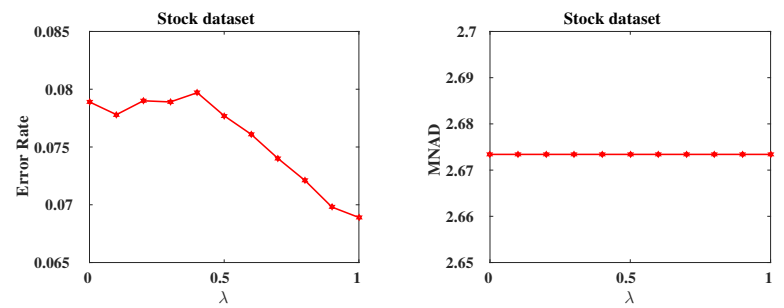

(c) Error Rate for the Stock (d) MNAD for the Stock dataset dataset

Figure 2: Performance w.r.t. influence ratio $\lambda$ on the two real-world datasets.

Impact of Global Influence Factor $\lambda$ : Global influence factor $\lambda$ is used to adjust how much a source gets influenced by its related sources when it provides a specific claim. In order to better demonstrate the effect of $\lambda$, we show the variation of Error rate and MNAD with different values of influence parameter $\lambda$ in Fig. 2. From Fig. 2, we can observe that the proposed IATD model performs differently with respect to the values of $\lambda$ on the two datasets. The different trends imply that the datasets may have different correlation patterns (which is also suggested by Fig. 3). Based on the results from Fig. 2, a relatively large $\lambda$ (close to 1) generally gives better results. This indicates that if two sources are correlated, they may influence each other strongly. These experiments illustrate that to enhance the performance of truth discovery, inter-source influences need to be utilized properly.

\subsection{Experiments on Synthetic Data}

In order to demonstrate the advantages of our proposed model comprehensively, we conduct experiments on simulated dataset.

Simulation Settings: Each synthetic dataset contains 10000 entities, where 7000 entities are numerical and 3000 entities are categorical. Different levels of noise are added to the ground truth to simulate sources with different levels of trustworthiness. In this section, we set the trustworthiness of a source to be consistent when it generates numerical and categorical claims. Specifically, we generate 10 high-quality sources $\left(\sigma^{2}=1\right)$ and 10 low-quality sources $\left(\sigma^{2}=10\right)$. For a specific source $s$, when simulating numerical claims, we sample the noise from a Gaussian distribution. The mean of this Gaussian distribution is zero and the deviation is set
Correlation matrix on Flight dataset

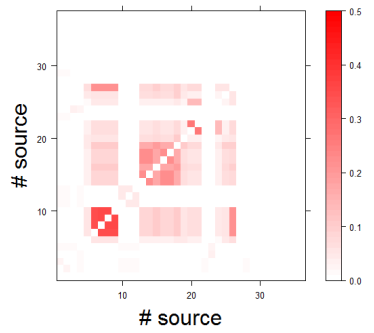

Correlation matrix on Stock dataset

Figure 3: Visualization of source correlations on the Flight and Stock dataset. Squares of darker color indicate strong correlations.

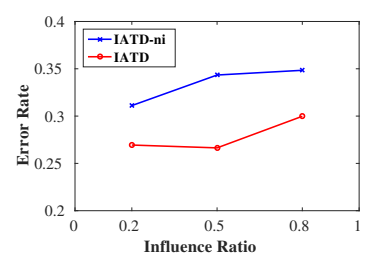

(a) Error Rate for Scenario 1

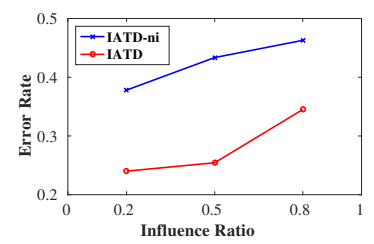

(c) Error Rate for Scenario 2

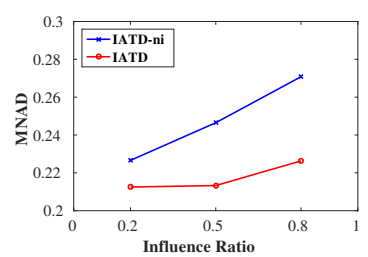

(b) MNAD for Scenario 1

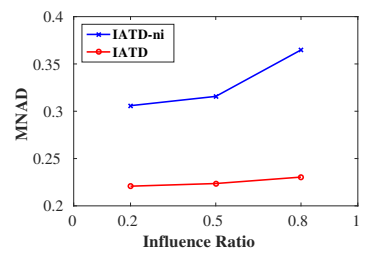

(d) MNAD for Scenario 2
Figure 4: Comparison of performance on the two scenarios. Red line and blue line demonstrate the performance of IATD and IATD-ni respectively.

based on the level of source trustworthiness. When simulating categorical claims, we first sample a factor $z$ from the same Gaussian distribution, and then compare it with a confidence threshold $\delta$. If $|z| \geq \delta$, we assign a random false choice to the claim; otherwise, the correct choice is assigned to the claim.

Two scenarios are considered in this experiment. For both scenarios, we test the proposed method with three levels of dependency. That is, we randomly allocate $[20 \%, 50 \%, 80 \%]$ of all the sources as independent sources, with others as influenced sources. For the influenced sources, we consider two scenarios with different ratios of influenced claims. For Scenario 1, the influenced sources provide $20 \%$ of their claims independently. For Scenario 2, the influenced sources provide $80 \%$ of their claims independently. Such settings are based on the Pareto Law ${ }^{2}$. The task for this section is to estimate the true value for each entity in the dataset.

Results and Discussions: We choose IATD-ni as the baseline method for this part to demonstrate the effectiveness of utilizing inter-source influences in truth discovery. The re-

\footnotetext{
${ }^{2}$ For many events, roughly $80 \%$ of the effects come from $20 \%$ of the causes.
} 
sults on the synthetic datasets are shown in Fig. 4. From the figures we can see that IATD method works consistently better than IATD-ni regardless the ratios of influences among sources and claims. Comparing the two scenarios, the results provided by IATD method are similar, while IATD-ni suffers a bigger performance degradation when there are more influenced claims. This again proves the importance of utilizing influences in truth discovery tasks, and demonstrates that the proposed method successfully models the source correlations and influences.

\section{RELATED WORK}

We investigate the related work from two aspects: truth discovery and information trustworthiness analysis.

Truth Discovery: Many existing truth discovery approaches are proposed to solve the problem of multi-source data aggregation based on source reliability estimation. Those approaches assume that if a source provides many trustworthy claims, this source is reliable, and if a claim is supported by many reliable sources, this claim is more trustworthy. Typically, they iteratively calculate source reliability and claim trustworthiness. Most of these approaches [2, 3, 7, 23, 25] make the assumption that sources make their claims independently. The detailed descriptions of these approaches can be found in Section 4.1.

There is some truth discovery work $[2,16,17,19]$ that takes source correlation into consideration. In $[2,16]$, source correlations are inferred based on the intuition that "if two sources provide the same false values, it is very likely that one copies from the other". However, these models do not precisely demonstrate how potential correlation can impact the estimation of sources' trustworthiness, and cannot directly handle data of numerical type. In [17] Qi et. al. propose a probabilistic model, which reveals the latent group structure among dependent sources. Different from our method, this approach assigns source weights at the group level instead of individual level. In the field of social sensing, Wang et. al. [19] propose Apollo to determine the correctness of reported observations in social media, considering both source reliabilities and correlations. However, their problem settings are different from ours. Apollo can only be used for binary claims (e.g. an event exists or not), and cannot be directly used in general truth discovery contexts.

The following truth discovery methods take different problem settings from ours. In [24], the authors adopt a semisupervised graph learning approach to improve the accuracy of truth estimation. In [6,22], confidence-aware methods are proposed for data with the long-tail phenomenon. In [12], the authors propose a fine-grained truth discovery model that utilizes the text information of claims and entities. The methods in $[10,27]$ extend truth discovery approaches to streaming data. There is also work $[16,20,26]$ proposed for the scenario that there is more than one truth for each entity. Since their problem settings are different ours, these methods are not compared in the experiments.

Information Trustworthiness Analysis: Our work is also related to the field of information trustworthiness analysis. Considerable efforts have been made for evaluating the reputation or trustworthiness of websites, users, and sources $[4,5,13]$. Unlike truth discovery methods, information trustworthiness analysis approaches are usually based on link analysis or based on explicit features extracted from the data.

\section{CONCLUSIONS}

As an emerging topic, truth discovery has shown a great potential in a wide range of applications thanks to its ability to estimate the truths and source trustworthiness simultaneously. Many existing truth discovery methods assume that sources make claims independently, which may be violated in real world, as source correlations are ubiquitous. For those methods who do consider source correlations, they limit the claims to be categorical type. To better fit the real world applications, in this paper, we propose a probabilistic model that can handle both challenges. By taking the source correlations as prior knowledge for influence derivation, the proposed influence-aware truth discovery model can estimate the trustworthiness of a source more accurately. Moreover, claims of both numerical and categorical types are modeled in a unified manner. Experimental results on two real world datasets prove the effectiveness of the proposed IATD model. Furthermore, experimental results on the simulated datasets illustrate the nice properties of the proposed IATD model under different scenarios.

\section{ACKNOWLEDGMENTS}

This work was sponsored in part by US National Science Foundation under grant IIS-1319973, IIS-1553411 and CNS1566374. The views and conclusions contained in this paper are those of the authors and should not be interpreted as representing any funding agency.

\section{REFERENCES}

[1] A. P. Dawid and A. M. Skene. Maximum likelihood estimation of observer error-rates using the EM algorithm. Applied statistics, pages 20-28, 1979.

[2] X. L. Dong, L. Berti-Equille, and D. Srivastava. Integrating conflicting data: the role of source dependence. PVLDB, 2(1):550-561, 2009.

[3] A. Galland, S. Abiteboul, A. Marian, and P. Senellart. Corroborating information from disagreeing views. In Proc. of WSDM, 2010.

[4] H. Ge, J. Caverlee, and H. Lu. Taper: A contextual tensor-based approach for personalized expert recommendation. In Proc. of RecSys, 2016.

[5] A. Gupta and P. Kumaraguru. Credibility ranking of tweets during high impact events. In Proc. of PSOSM Workshop, pages 2:2-2:8, 2012.

[6] Q. Li, Y. Li, J. Gao, L. Su, B. Zhao, M. Demirbas, W. Fan, and J. Han. A confidence-aware approach for truth discovery on long-tail data. PVLDB, 8(4):425-436, 2014.

[7] Q. Li, Y. Li, J. Gao, B. Zhao, W. Fan, and J. Han. Resolving conflicts in heterogeneous data by truth discovery and source reliability estimation. In Proc. of SIGMOD, 2014.

[8] X. Li, X. L. Dong, K. Lyons, W. Meng, and D. Srivastava. Truth finding on the deep web: is the problem solved? PVLDB, 6(2):97-108, 2012.

[9] X. Li, X. L. Dong, K. B. Lyons, W. Meng, and D. Srivastava. Scaling up copy detection. In Proc. of ICDE, 2015.

[10] Y. Li, Q. Li, J. Gao, L. Su, B. Zhao, W. Fan, and J. Han. On the discovery of evolving truth. In Proc. of SIGKDD, 2015. 
[11] C.-b. Lin. Projected gradient methods for nonnegative matrix factorization. Neural computation, 19(10):2756-2779, 2007.

[12] F. Ma, Y. Li, Q. Li, M. Qiu, J. Gao, S. Zhi, L. Su, B. Zhao, H. Ji, and J. Han. Faitcrowd: Fine grained truth discovery for crowdsourced data aggregation. In Proc. of SIGKDD, 2015.

[13] L. Page, S. Brin, R. Motwani, and T. Winograd. The pagerank citation ranking: Bringing order to the web. 1999.

[14] J. Pasternack and D. Roth. Making better informed trust decisions with generalized fact-finding. In Proc. of IJCAI.

[15] J. Pasternack and D. Roth. Latent credibility analysis. In Proc. of $W W W, 2013$.

[16] R. Pochampally, A. Das Sarma, X. L. Dong, A. Meliou, and D. Srivastava. Fusing data with correlations. In Proc. of SIGMOD, 2014.

[17] G.-J. Qi, C. C. Aggarwal, J. Han, and T. Huang. Mining collective intelligence in diverse groups. In Proc. of $W W W, 2013$.

[18] A. D. Sarma, X. L. Dong, and A. Halevy. Data integration with dependent sources. In Proc. of EDBT, 2011.

[19] D. Wang, M. T. Amin, S. Li, T. Abdelzaher, L. Kaplan, S. Gu, C. Pan, H. Liu, C. C. Aggarwal, R. Ganti, et al. Using humans as sensors: an estimation-theoretic perspective. In Proc. of IPSN, 2014.

[20] X. Wang, Q. Z. Sheng, X. S. Fang, L. Yao, X. Xu, and X. Li. An integrated bayesian approach for effective multi-truth discovery. In Proc. of CIKM, 2015.

[21] J. Whitehill, T.-f. Wu, J. Bergsma, J. R. Movellan, and P. L. Ruvolo. Whose vote should count more: Optimal integration of labels from labelers of unknown expertise. In Proc. of NIPS, 2009.

[22] H. Xiao, J. Gao, Q. Li, F. Ma, L. Su, Y. Feng, and A. Zhang. Towards confidence in the truth: A bootstrapping based truth discovery approach. In Proc. of SIGKDD, 2016.

[23] X. Yin, J. Han, and P. S. Yu. Truth discovery with multiple conflicting information providers on the web. TKDE, 20(6):796-808, 2008.

[24] X. Yin and W. Tan. Semi-supervised truth discovery. In Proc. of $W W W, 2011$.

[25] B. Zhao and J. Han. A probabilistic model for estimating real-valued truth from conflicting sources. In Proc. of QDB, 2012.

[26] B. Zhao, B. I. Rubinstein, J. Gemmell, and J. Han. A bayesian approach to discovering truth from conflicting sources for data integration. PVLDB, 5(6):550-561, 2012.

[27] Z. Zhao, J. Cheng, and W. Ng. Truth discovery in data streams: A single-pass probabilistic approach. In Proc. of CIKM, 2014.

\section{APPENDIX}

\section{A. DETAILED DERIVATIONS IN M-STEP}

The detailed expressions of the partial derivatives for entities with categorical and numerical values are as follows:
Entities with Categorical Values: For entities with categorical values, if the source is influenced, the partial derivatives of $\sigma_{s}$ and $\sigma_{j}$ and $b_{v}$ are calculated using:

$$
\begin{aligned}
& \frac{\partial \mathcal{L}_{s, v}}{\partial \sigma_{s}}=-\left(h\left(-\epsilon_{s, v}+b_{v}+g^{(c)}\right)-\delta\left(c_{s, v}, t_{v}\right)\right)(1-\lambda) \\
&+\frac{1}{\left|C_{s, \cdot}\right|}\left(\frac{2\left(1+\alpha_{e}\right)}{\sigma_{s}}-2 \beta_{e} \sigma_{s}^{-3}\right), \\
& \frac{\partial \mathcal{L}_{s, v}}{\partial \sigma_{j}}=-\left(h\left(-\epsilon_{s, v}+b_{v}+g^{(c)}\right)-\delta\left(c_{s, v}, t_{v}\right)\right) \frac{\lambda}{\left|A_{s, v}\right|}, \\
& \frac{\partial \mathcal{L}_{s, v}}{\partial b_{v}}=h\left(-\epsilon_{s, v}+b_{v}+g^{(c)}\right)-\delta\left(c_{s, v}, t_{v}\right)+\frac{1}{|C \cdot, v|} \frac{\left(b_{v}-\mu_{b}\right)}{\sigma_{b}^{2}} .
\end{aligned}
$$

If the source makes the claim independently, the partial derivatives of $\sigma_{s}$ and $b_{v}$ are:

$$
\begin{aligned}
\frac{\partial \mathcal{L}_{s, v}}{\partial \sigma_{s}}= & -\left(h\left(-\epsilon_{s, v}+b_{v}+g^{(c)}\right)-\delta\left(c_{s, v}, t_{v}\right)\right) \\
& +\frac{1}{\left|C_{s, \cdot}\right|}\left(\frac{2\left(1+\alpha_{e}\right)}{\sigma_{s}}-2 \beta_{e} \sigma_{s}^{-3}\right), \\
\frac{\partial \mathcal{L}_{s, v}}{\partial b_{v}}= & h\left(-\epsilon_{s, v}+b_{v}+g^{(c)}\right)-\delta\left(c_{s, v}, t_{v}\right)+\frac{1}{\left|C_{,, v}\right|} \frac{\left(b_{v}-\mu_{b}\right)}{\sigma_{b}^{2}} .
\end{aligned}
$$

Entities with Numerical Values: For entities with numerical values, if the source is influenced, the partial derivatives of $\sigma_{s}, \sigma_{j}$ and $b_{v}$ are defined as:

$$
\begin{aligned}
\frac{\partial \mathcal{L}_{s, v}}{\partial \sigma_{s}}= & (1-\lambda)\left(-\frac{\left(c_{s, v}-t_{v}\right)^{2}}{\left(b_{v}+\epsilon_{s, v}+g^{(n)}\right)^{3}}+\frac{1}{b_{v}+\epsilon_{s, v}+g^{(n)}}\right) \\
& +\frac{1}{\left|C_{s, \cdot}\right|}\left(\frac{2\left(1+\alpha_{e}\right)}{\sigma_{s}}-2 \beta_{e} \sigma_{s}^{-3}\right), \\
\frac{\partial \mathcal{L}_{s, v}}{\partial \sigma_{j}}= & \frac{\lambda}{\left|A_{s, v}\right|}\left(-\frac{\left(c_{s, v}-t_{v}\right)^{2}}{\left(b_{v}+\epsilon_{s, v}+g^{(n)}\right)^{3}}+\frac{1}{b_{v}+\epsilon_{s, v}+g^{(n)}}\right), \\
\frac{\partial \mathcal{L}_{s, v}}{\partial b_{v}}= & -\frac{\left(c_{s, v}-t_{v}\right)^{2}}{\left(b_{v}+\epsilon_{s, v}+g^{(n)}\right)^{3}}+\frac{1}{b_{v}+\epsilon_{s, v}+g^{(n)}} \\
& +\frac{1}{\left|C_{,, v}\right|} \frac{\left(b_{v}-\mu_{b}\right)}{\sigma_{b}^{2}} .
\end{aligned}
$$

If the source makes the claim independently, the partial derivatives of $\sigma_{s}$ and $b_{v}$ are:

$$
\begin{aligned}
\frac{\partial \mathcal{L}_{s, v}}{\partial \sigma_{s}}= & -\frac{\left(c_{s, v}-t_{v}\right)^{2}}{\left(b_{v}+\epsilon_{s, v}+g^{(n)}\right)^{3}}+\frac{1}{b_{v}+\epsilon_{s, v}+g^{(n)}} \\
& +\frac{1}{\left|C_{s, \cdot}\right|}\left(\frac{2\left(1+\alpha_{e}\right)}{\sigma_{j}}-2 \beta_{e} \sigma_{j}^{-3}\right), \\
\frac{\partial \mathcal{L}_{s, v}}{\partial b_{v}}= & -\frac{\left(c_{s, v}-t_{v}\right)^{2}}{\left(b_{v}+\epsilon_{s, v}+g^{(n)}\right)^{3}}+\frac{1}{b_{v}+\epsilon_{s, v}+g^{(n)}} \\
& +\frac{1}{\left|C_{,, v}\right|} \frac{\left(b_{v}-\mu_{b}\right)}{\sigma_{b}^{2}} .
\end{aligned}
$$

